\title{
FDTD simulation of electromagnetic wave propagation in magnetic randomly inhomogeneous media
}

This paper was downloaded from TechRxiv (https://www.techrxiv.org).

\section{LICENSE}

CC BY 4.0

SUBMISSION DATE / POSTED DATE

$30-09-2021 / 01-11-2021$

\section{CITATION}

Makarov, Pavel; Ustyugov, Vladimir; Kotov, Leonid; Nekipelov, Sergey V.; Sivkov, Viktor (2021): FDTD simulation of electromagnetic wave propagation in magnetic randomly inhomogeneous media. TechRxiv. Preprint. https://doi.org/10.36227/techrxiv.16709116.v2

DOI 


\title{
FDTD simulation of electromagnetic wave propagation in magnetic randomly inhomogeneous media
}

\author{
Pavel Makarov ${ }^{1,2}$, Vladimir Ustyugov ${ }^{1}$, Leonid Kotov ${ }^{1}$, Sergey Nekipelov ${ }^{2}$ and Viktor Sivkov ${ }^{2}$ \\ ${ }^{1}$ Syktyvkar State University named after Pitirim Sorokin, Syktyvkar, Russia \\ ${ }^{2}$ Institute of Physics and Mathematics, Komi Science Center Ural Division of the Russian Academy of Sciences, \\ Syktyvkar, Russia
}

\begin{abstract}
An algorithm for the numerical simulation of the propagation of electromagnetic waves in randomly inhomogeneous magnetic media by the FDTD method has been developed. The formulated algorithm is suitable for analyzing the main timing characteristics, as well as identifying the features of the propagation of various types of signals in both time-independent and time-dependent layered randomly inhomogeneous media. The simulation of the propagation of the sine pulse, sine signal and square wave in time-independent magnetic randomly inhomogeneous media with a various levels of phase contrast of two types - with a "diffuse" distribution of inhomogeneities and their "close packing" is carried out. The influence of the concentration of magnetic granules and the type of their distribution on the characteristics of the transmitted and reflected signals is revealed.
\end{abstract}

Index Terms-Electromagnetics, Magnetic films, Numerical methods

\section{INTRODUCTION}

$\mathbf{T}$ HE model of a randomly inhomogeneous medium has been in great demand for many years both in fundamental science and in applied research and engineering applications [1], [2]. There are specific problems of statistical radiophysics and acoustics, in which this model plays an important role. Similar problems and its solution methods arise in plasma physics, solid state physics, magnetohydrodynamics and other areas [3], [4]. Furthemore, widespread use of optical and acoustic diagnostic methods in modern medicine is also based on the features of electromagnetic wave propagation in stochastic systems [5].

In the literature cited above [1]-[5], the problem of signal propagation in randomly inhomogeneous media is solved by various semi-analytical, qualitative methods, which requires very significant efforts to develop an appropriate model and establish the limitations of its applicability. In addition, these approximate methods can only give a certain averaged picture of the phenomenon, which differs dramatically from the possible implementation in each specific situation.

In this regard, it is important to development of numerical methods that make it possible to visually and simply represent the process of signal propagation in specific stochastic media. Currently, there are many similar techniques, but the most common are grid methods. They are based on the procedure for discretizing space-time and the transition from differential equations for continuous functions to finite-difference equations for functions of discrete variables. The fundamental work in this area was the Kane Yee paper [6], in which the method of Finite Differences in the Time Domain (FDTD) was first described. This work did not receive a wide response in the scientific community due to high memory usage and extraordinary computer time required for implementation, but recently the FDTD method is experiencing a "rebirth" [7], [8].

Manuscript received September 15, 2021; revised ???????, 2021. Corresponding author: P. Makarov (email: makarovpa@syktsu.ru).
There are many scientific papers devoted to the application of the FDTD method for solving electrodynamic problems in randomly inhomogeneous media [9]-[21]. Nevertheless, this area not only has not exhausted itself, but on the contrary it continues to remain very attractive, as it can potentially stimulate the study of many interesting effects.

In particular, the development of an effective FDTD algorithm for modeling the propagation of electromagnetic waves in randomly inhomogeneous media can be useful for solving the following problems:

- determination of the laws of light propagation in composite nanostructured materials;

- investigation of the dynamic characteristics of materials containing various inclusions or having structure defects;

- quantification of interference arising from the passage of a radio signal in a non-stationary inhomogeneous environment under adverse weather conditions.

In the vast majority of cases the authors develop the FDTD method for modeling the properties of media with inhomogeneous permittivity and conductivity. In the course of our research, we were engaged in the development of a method suitable for modeling the electrodynamics of magnetic non-homogeneous nanocomposite structures. In our opinion, there are considerably fewer papers published in this field. As an exception, we can mention the article [22], in which the FDTD method was applied to calculate propagation constants in waveguide containing ferrites. There are also works [17], [18] in which stochastic S-FDTD methods applied to Cartesian problems are developed. Despite its benefits, though, there are still concerns to be resolved, like the dispersion errors and curved interfaces in the vicinity of electrically-large nanostructured devices [21]. All these papers also do not address the magnetic properties of the medium. The works [15], [19] consider the application of S-FDTD methods specifically to the case of electromagnetic wave propagation in magnetized ionosphere plasma. 
Thus, the aim of this work is to develop a simple and effective FDTD algorithm to demonstrate some features of the propagation of electromagnetic waves in randomly inhomogeneous magnetic nanocomposites. We focus our attention mainly on the reflecting and transmitting properties. In this paper, we restrict ourselves to the simplest case of one-dimensional layered inhomogeneous systems, which, of course, will not give an opportunity to study all the electrodynamic properties, but, nevertheless, can serve as a starting point for more complex realizations.

Objectives of our research are

- obtaining the finite-difference approximation of Maxwells equations;

- the convergence testing of the developed algorithm at different wavelengths;

- simulation of reflecting and transmission some types of signals in composites with different types of magnetic inhomogeneity.

\section{BRIEF THEORY}

As is known [23], macroscopic Maxwell's equations have the form

$$
\begin{gathered}
\nabla \times \mathbf{E}=-\frac{\partial \mathbf{B}}{\partial t}, \quad \nabla \times \mathbf{H}=\mathbf{J}_{\mathrm{ext}}+\frac{\partial \mathbf{D}}{\partial t}, \\
\nabla \cdot \mathbf{D}=\rho_{\mathrm{ext}}, \quad \nabla \cdot \mathbf{B}=0 .
\end{gathered}
$$

With the system of constitutive equations

$$
\mathbf{D}=\varepsilon \varepsilon_{0} \mathbf{E}, \quad \mathbf{B}=\mu \mu_{0} \mathbf{H}
$$

the equations (1) and (2) completely determine all the electrodynamic properties of the system.

We will consider the system in the absence of external charges $\rho_{\mathrm{ext}}=0$, for which $\mathbf{J}_{\mathrm{ext}}, \varepsilon$ and $\mu$ are given functions of space-time variables. In this case, the mathematical description of the problem is reduced to the laws of Faraday and Ampere (1) and material relations (3).

Next, we will investigate the propagation of an electromagnetic field along the direction of the Cartesian axis $O x$ in isotropic magnetic material $\varepsilon=\varepsilon(\mathbf{r}, t), \mu=\mu(\mathbf{r}, t)$, when $\mathbf{J} \| O z$.

It is clearly from our assumptions, that $\mathbf{E}=E_{z} \mathbf{e}_{z}$, $\mathbf{H}=H_{y} \mathbf{e}_{y}$ and the main system of the equations (1) and (3) takes the form

$$
\frac{\partial E_{z}}{\partial x}=\mu_{0} \frac{\partial\left(\mu H_{y}\right)}{\partial t}, \quad \frac{\partial H_{y}}{\partial x}=J_{z}+\varepsilon_{0} \frac{\partial\left(\varepsilon E_{z}\right)}{\partial t} .
$$

So, our task is reduced to the study of the dynamics of two scalar functions $E_{z}(x, t)$ and $H_{y}(x, t)$, related by the equations (4). In this case, all the electrodynamic properties of the medium are completely determined by the stochastic functions $\varepsilon(\mathbf{r}, t)$ and $\mu(\mathbf{r}, t)$.

At the next step we discretize space-time by introducing the following point functions

$$
f(x, t)=f(m \Delta x, q \Delta t)=f^{q}[m] .
$$

After that, we choose two grids for the electric $E_{z}^{q}[m]$ and magnetic $H_{y}^{q}[m]$ fields, shifted relative to each other in a checkerboard pattern, as suggested in the classical work [6].
This allows us to write the finite-difference analogue of the equations (4) in the form

$$
\begin{aligned}
& H_{y}^{q+\frac{1}{2}}\left[m+\frac{1}{2}\right]= \\
& =\frac{1}{\mu^{q+\frac{1}{2}}\left[m+\frac{1}{2}\right]}\left\{\mu^{q-\frac{1}{2}}\left[m+\frac{1}{2}\right] H_{y}^{q-\frac{1}{2}}\left[m+\frac{1}{2}\right]+\right. \\
& \left.+\frac{\Delta t}{\mu_{0} \Delta x}\left(E_{z}^{q}[m+1]-E_{z}^{q}[m]\right)\right\}, \\
& E_{z}^{q+1}[m]=\frac{1}{\varepsilon^{q+1}[m]}\left\{\varepsilon^{q}[m] E_{z}^{q}[m]-\Delta t J_{z}^{q+\frac{1}{2}}[m]+\right. \\
& \left.+\frac{\Delta t}{\varepsilon_{0} \Delta x}\left(H_{y}^{q+\frac{1}{2}}\left[m+\frac{1}{2}\right]-H_{y}^{q+\frac{1}{2}}\left[m-\frac{1}{2}\right]\right)\right\},
\end{aligned}
$$

The equations (6) and (7) make it possible to implement the iterative Yee algorithm [6]-[8], which determines the entire dynamics of the electromagnetic field in the considered case of a isotropic randomly inhomogeneous medium. The obtained equalities differ from the well-known analogs of [10], [11], [13], [14], [16], [17] in that they allow describing dynamics of inhomogeneous layered structures such as magnetic nanocomposite films. Moreover, developed algorithm is applicable for calculation the dynamics not only for time-independent, but also for time-dependent stochastic systems. At the same time, it is obvious that the decisive role in the success of the implementation of the formulated method is played by the "correctly selected" parameters of the space-time grid. In any case, a necessary condition for the operation of the algorithm is the inequalities

$$
\Delta x \ll \lambda, \quad \Delta t \ll 1 / \nu,
$$

where $\lambda$ - wavelength, and $\nu$ - frequency.

\section{Simulation SCheme}

To demonstrate the efficiency of the developed method, a numerical experiment was carried out in accordance with the scheme shown in Fig. 1. As follows from Fig. 1, a sequence of three layers is considered: 1 and $3-$ semi-infinite airspaces ( $\varepsilon=\mu=1$ ) surrounding a randomly heterogeneous medium 2 with a total length of $L$. In the medium 1 there are two antennas: transmitting $A_{\mathrm{s}}$ and receiving $A_{\mathrm{r}}$. Antenna $A_{\mathrm{s}}$ generates a unidirectional signal propagating towards the medium 2 . Antenna $A_{\mathrm{t}}$, located in medium 3 , receives a signal that has passed through medium 2; $A_{\mathrm{r}}$ - catches the reflected signal.

The main geometrical parameters of the scheme are also shown in Fig. 1. The interface between the media 1 and 2 is at a distance of $L_{\mathrm{s}}$ from the signal source $A_{\mathrm{s}}$, and the receiving antenna $A_{\mathrm{t}}$ is at a distance of $L_{\mathrm{t}}$ from the interface 2 and 3. Receiver $A_{\mathrm{r}}$ is located to the left of the source at a distance of $L_{\mathrm{r}}$. It is clearly, how this parameters affect the time delays $\tau_{\mathrm{r}}$ and $\tau_{\mathrm{t}}$ of the appearance of signals on the corresponding antennas.

Thus, there is only a reflected signal in the $x<L_{\mathrm{r}}$ area; $x>L_{\mathrm{s}}+L-$ only transmitted signal; in the interval $L_{r} \leqslant x \leqslant L_{\mathrm{s}}+L$, according to the superposition principle, there is a total field, which is the sum of the incident and scattered waves. The software implementation of the proposed 


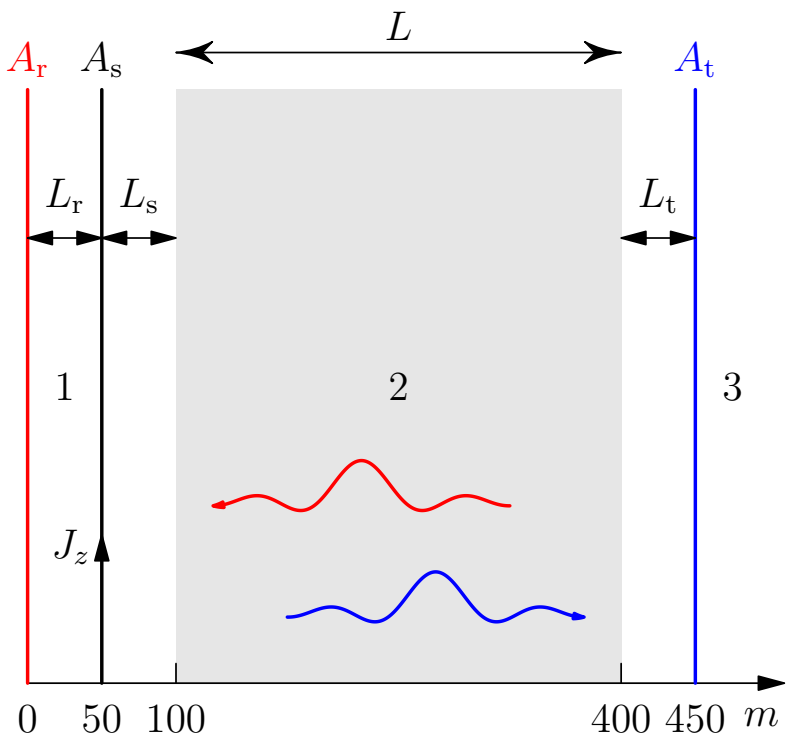

Fig. 1. Numerical experiment scheme: 1 and 3 - semi-infinite air spaces surrounding a randomly inhomogeneous medium $2 ; A_{\mathrm{s}}-$ signal source antenna; $A_{\mathrm{r}}-$ receiving antenna registering the reflected signal; $A_{\mathrm{t}}$ antenna receiving the signal transmitted through the medium 2 .

scheme takes this into account using a technique known as TF/SF (Total-Field/Scattered Field) [7], [8].

Medium 2 is assumed to be non-conductive, multicomponent and weakly inhomogeneous, so that the main material of the medium (filling matrix) has a relative permittivity $\varepsilon_{\mathrm{m}}$. The components that form the magnetic inhomogeneities of the medium 2 are modeled by a set of segments of length $d_{j}^{(i)} \sim L^{-1}$ with magnetic permeability $\mu_{j}$ and relative linear concentration $n_{j}$ such that

$$
\sum_{i} d_{j}^{(i)}=n_{j} \cdot L, \quad \sum_{j=1}^{N} n_{j} \leqslant 1, \quad \sum_{j=1}^{N} \mu_{j} n_{j}=\mu_{\mathrm{eff}},
$$

where $N-$ is the total number of components, that form magnetic inhomogeneities of the medium 2. In our simulation, we neglected the time-varying properties of the medium 2 , although the developed algorithm potentially allows to take this into account.

\section{Simulation RESUlts}

During the simulation, according to the algorithm developed in this work, time diagrams of various types of signals were built on the transmitting $A_{\mathrm{s}}$ and receiving antennas $A_{\mathrm{r}}, A_{\mathrm{t}}$ for different randomly inhomogeneous magnetic nanocomposite films.

In all calculations, the relative permittivity of the filling matrix was assumed to be $\varepsilon_{\mathrm{m}}=4$. An example of the distribution of material parameter values over the film thickness in the case of a composition of two magnetic components $(N=2)$ with significantly different magnetic permeabilities $\mu_{1} \gg \mu_{2}$ and a total concentration $n=n_{1}+n_{2}=0.1\left(n_{1}=n_{2}\right)$ is shown in Fig. 2. Here are the graphs for randomly inhomogeneous magnetic nanocomposite films of two types: with a random distribution of inhomogeneities over the entire thickness of the film $(a)$ and their "close packing" in the center of film $(b)$. (a)

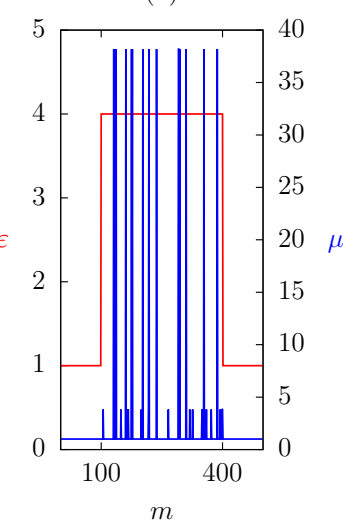

$(b)$

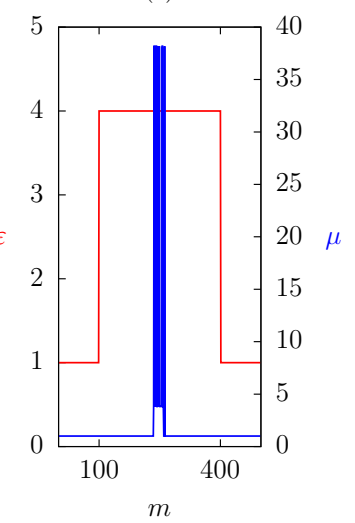

Fig. 2. Distribution of material parameters over the thickness of the film in the case of a dielectric matrix with two magnetic components significantly different magnetic permeabilities $\mu_{1} \gg \mu_{2}$ and total concentration $n=0.1$.

For any concentration values of inhomogeneities $n_{j}$, the values $\mu_{j}$ were selected so that the effective magnetic permeability of the medium 2 was always the same and equal to $\mu_{\text {eff }}=3$. So, the effective film impedance in all simulations was chosen slightly lower than the air impedance $\rho_{\mathrm{eff}}=\rho_{0} \sqrt{\mu_{\mathrm{eff}} / \varepsilon_{\mathrm{m}}} \approx 0.87 \rho_{0}$.

Further, we first of all studied the area of applicability of the developed algorithm. In the series of numerical simulations, it was proved that the program implementation gives excellent results in all cases when the wavelength of the signal $\lambda \gtrsim L$. It was also shown that the region $\lambda \ll L$ is not available for modeling according to the discussed algorithm. For greater clarity, all calculations in this article are given for the case when $\lambda \sim L$.

\section{A. Sine pulse}

In order to neglect interference effects and to simplify the propagation picture as much as possible, in the first part of the study was examined the propagation of the short sine pulse (with a pulse duration equal to the period of the sunusoidal signal).

Fig. 3 shows curves, illustrating the time dependence of the signal received by the antenna $A_{\mathrm{r}}$. For constructing this figure, the medium 2 was modelled by a dielectric matrix with two types $(N=2)$ of magnetic granules included into it. The red and purple curves correspond to different concentrations of granules $n=0.1$ and $n=0.4$ (the magnetic permeabilities for these cases were chosing so that in both cases $\mu_{\text {eff }}=3$ ). The solid curves in this and all the following figures correspond to the case of a "diffuse" distribution of inhomogeneities over the entire film thickness; dashed lines illustrate to the case of "close packing" of granules. In addition, the black curve in Fig. 3 shows the reflected signal for a homogeneous medium with the same impedance.

As is known from classical electrodynamics [23], in the case of normal incidence of a wave from air on a homogeneous 


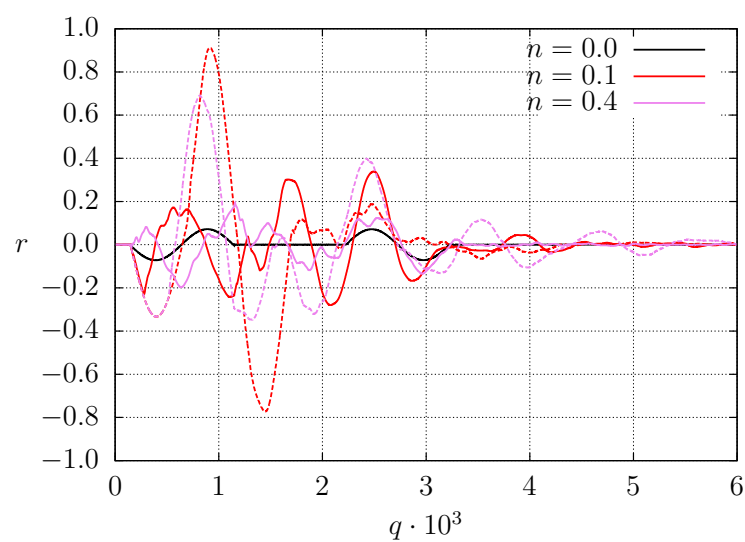

Fig. 3. Reflected sine pulses for various concentration of magnetic inclusions.

medium with an impedance of $\eta$, the reflection coefficient for the parameters we have chosen should be small:

$$
r=\frac{\eta_{2}-\eta_{1}}{\eta_{1}+\eta_{2}}=\frac{0.87 \eta_{0}-\eta_{0}}{\eta_{0}+0.87 \eta_{0}} \approx-0.07 .
$$

The black line in Fig. 3 confirms the validity of the result (10). The emergence of two signals on the antenna $A_{\mathrm{r}}$ that are in antiphase with respect to each other is associated with signal reflection from both boundaries of the medium 2 . Comparison of the red and purple curves with the black line in Fig. 3 shows that the presence of even a small number of magnetic irregularities can significantly change the reflection. For example, for the value $n=0.1$ the maximum value of the reflection coefficient increases more than tenfold for the case of "close packing" of granules and fivefold in the case of a "diffuse" distribution of inhomogeneities. At the same time, it can be seen that with an the rising of the magnetic granules concentration $n$, the phase contrast of the medium 2 is decreased, and the character of the reflection becomes more similar to the reflection from a homogeneous medium (this is especially noticeable in the case of a "diffuse packing" of granules).

Fig. 4 shows graphs, illustrating the time dependence of the signals transmitted through the medium 2 , received by the antenna $A_{\mathrm{t}}$. All parameters for which these curves are plotted are similar to those in Fig. 3. The study of Fig. 4 shows that the character and concentration of the magnetic inhomogeneity of the medium affects on the characteristics of the transmitted signal strongly less that on the parameters of reflected wave in the case of a "diffuse packing" of magnetic inclusions.

So, the analysis that we performed allows to conclude that FDTD simulation:

- gives an adequate picture of the propagation of sine pulses in randomly inhomogeneous magnetic composites in the entire range $\lambda \gtrsim L$;

- especially important when studying reflection from media with a strong phase contrast in the case of $\lambda \sim L$.

\section{B. Sine signal}

Now, after we have studied in detail the propagation of a single sine pulse in a magnetic randomly inhomogeneous

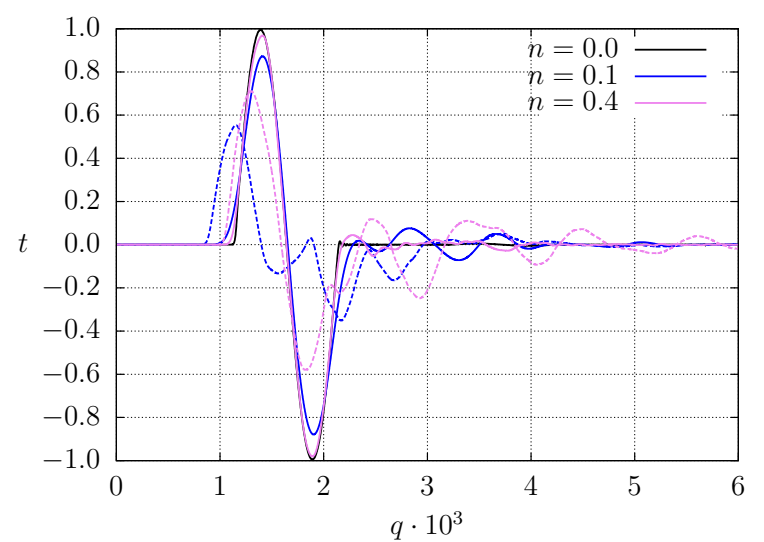

Fig. 4. Transmitted sine pulses for various concentration of magnetic inclusions.

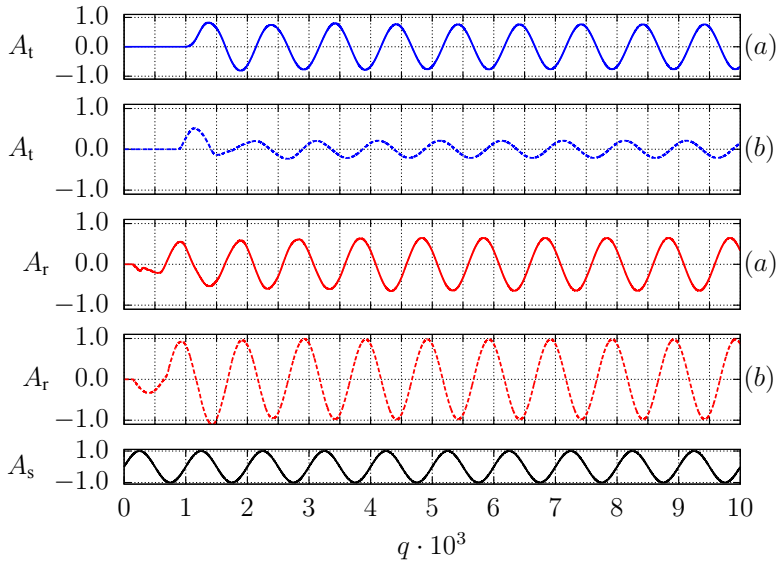

Fig. 5. Timing diagrams of the signals on the transmitting and receiving antennas in the case of a sine signal propagation through the media 2 with the parameters identically to Fig. 2.

medium, we can proceed to the analysis of wave dynamics. In this subsection, we investigate the simplest possible case of a sinusoidal signal. Fig. 5 demonstrate timing diagrams of the signals on the transmitting and receiving antennas in the case of a sine signal propagation through magnetic inhomogeneous media 2 consists of two magnetic components with significantly different magnetic permeabilities $\mu_{1} \gg \mu_{2}$. All parameters to construct the Fig. 5 is chosen identically to Fig. 2.

From the Fig. 5 we can see, that regardless of the type of packing of inclusions $((a)$ or $(b))$, a stationary mode is quickly established on the receiving antennas. The time of establishing a stable mode with our conditions is approximately one signal period. Mainly, the curves in Fig. 5 confirm facts, that was already discussed in the analysis of Figs. 3 and 4. Additionally, we can conclude that the type of granule packing $((a)$ or $(b))$ under the same conditions dramatically affects the reflection and transmission coefficients. So, for the case of a random distribution of inhomogeneities over the entire film thickness $(a)$, the greater part of the electromagnetic wave energy passes through the medium. However, in the case of $(b)$, everything happens exactly the opposite. It is pertinent to recall here that 


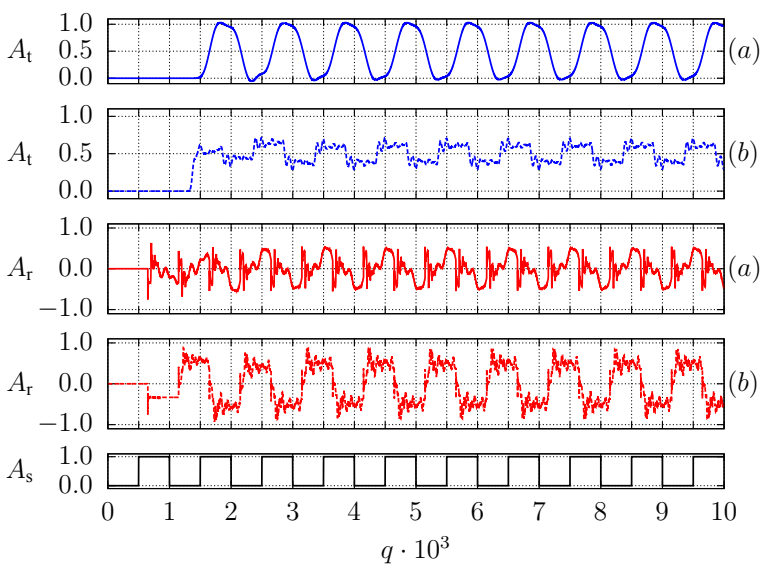

Fig. 6. Timing diagrams of the signals on the transmitting and receiving antennas in the case of a square wave propagation through the media 2 with the parameters identically to Figs. 2 and 5.

all the above conclusions refer to the case of sufficiently low concentrations of magnetic inclusions $n=0.1$.

\section{Square wave}

And in the end, we will show that the developed algorithm is useful for analyzing the propagation of signals of more complex types. Fig. 6 illustrates timing diagrams in the case of a square wave propagation with path parameters the same as into Fig. 2. It can be seen from it, that with the specified parameters of the path, the greater part of the electromagnetic wave passes through the film only in the case $(a)$. This result repeats what we already said in the discussion of Fig. 5, however, it is clearly seen that the shape of the transmitted and reflected signals is significantly distorted in comparison with the shape of the original wave. It is also interesting that for the considered model of a randomly inhomogeneous magnetic nanocomposite film, a reflected signal have a very significant amplitude (at the peak reaching values $A_{\mathrm{r}} \approx 0.6$ for case $(a)$ and $A_{\mathrm{r}} \approx 0.9$ for case $(b)$.

In addition, it is worth noting that in case $(b)$ the peak-topeak of the reflected signal is about ten times greater than the peak-to-peak of the transmitted signal. This circumstance can be very important, for example, for issues of digitizing analog signals.

\section{CONCLUSION}

In this work an algorithm for the numerical simulation of the propagation of electromagnetic waves in magnetic randomly inhomogeneous media by the FDTD method has been developed. That algorithm is suitable for analyzing the main time characteristics, as well as identifying the features of the propagation of various types of electromagnetic signals in layered randomly inhomogeneous magnetic media. The simulation of the signals propagation in stationary randomly inhomogeneous media with two types of phase contrast with a "diffuse" distribution of inhomogeneities and their "close packing" is carried out. Revealed influence concentration of inhomogeneities and the type of their distribution on the characteristics of the transmitted and reflected signals.

\section{ACKNOWLEDGMENT}

This study was supported by the Russian Science Foundation, project no. 21-72-20048.

\section{REFERENCES}

[1] Y. A. Kravtsov and A. I. Saichev, "Effects of double passage of waves in randomly inhomogeneous media," Sov. Phys. Usp., vol. 25, pp. 494-508, 1982.

[2] V. I. Klyatskin, "Electromagnetic wave propagation in a randomly inhomogeneous medium as a problem in mathematical statistical physics," Phys. Usp., vol. 47, pp. 169-186, 2004.

[3] A. M. Prokhorov, F. V. Bunkin, K. S. Gochelashvili, and S. V. I, "Propagation of laser radiation in randomly inhomogeneous media," Sov. Phys. Usp., vol. 18, pp. 826-847, 1975.

[4] V. L. Kuzmin and V. P. Romanov, "Coherent phenomena in light scattering from disordered systems," Phys. Usp., vol. 39, pp. 231-260, 1996.

[5] V. V. Tuchin, "Light scattering study of tissues," Phys. Usp., vol. 40, pp. 495-515, 1997.

[6] K. Yee, "Numerical solution of initial boundary value problems involving maxwell's equations in isotropic media," IEEE Trans. AP, vol. 14, no. 3, pp. 302-307, 1966.

[7] U. S. Inan and R. A. Marshall, Numerical electromagnetics. The FDTD method. Cambridge University Press, 2011.

[8] A. Taflove, A. Oskooi, and S. G. Johnson, Advances in FDTD computational electrodynamics photonics and nanotechnology. Artech House, 2013.

[9] S. G. Tanyer, M. Karaman, and I. Öztürk, "Analysis of wave propagation in inhomogeneous media using fdtd method and its applications," in MMET Conference Proceedings, 1998, pp. 629-631.

[10] C. D. Moss, F. L. Teixeira, Y. E. Yang, and J. A. Kong, "Finite-difference time-domain simulation of scattering from objects in continuous random media," IEEE Trans. GRS, vol. 40, no. 1, pp. 178-186, 2002.

[11] S. H. Tseng, J. H. Greene, A. Taflove, D. Maitland, V. Backman, and J. T. Walsh, "Exact solution of maxwells equations for optical interactions with a macroscopic random medium," Optics Letters, vol. 29, no. 12, pp. 1393-1395, 2004.

[12] R. A. Chilton and R. Lee, "The lobatto cell: robust, explicit, higher order fdtd that handles inhomogeneous media," IEEE Trans. AP, vol. 56, no. 8, pp. 2167-2177, 2008

[13] J. Li, L. X. Guo, H. Zeng, and X. B. Han, "Investigation of composite electromagnetic scattering from ship-like target on the randomly rough sea surface using fdtd method," Chinese Physics B, vol. 18, no. 7, pp. 2757-2763, 2009

[14] Y. Miyazaki and K. Kouno, "Fdtd analysis of spatial filtering of scattered waves for optical ct of medical diagnosis," IEEJ Trans. FM, vol. 129, no. 10, pp. 693-698, 2009.

[15] Y. Yu and J. J. Simpson, "An e-j collocated 3-d fdtd model of electromagnetic wave propagation in magnetized cold plasma," IEEE Trans. $A P$, vol. 58, no. 2, pp. 469-478, 2010.

[16] J. Li, L. X. Guo, H. Zeng, and X. B. Han, "Investigation on wideband scattering of a $2 \mathrm{~d}$ target above $1 \mathrm{~d}$ randomly rough surface by fdtd method," Optics Express, vol. 19, no. 2, pp. 1091-1100, 2011.

[17] T. Tan, A. Taflove, and V. Backman, "Single realization stochastic fdtd for weak scattering waves in biological random media," IEEE Trans. $A P$, vol. 61, no. 2, pp. 818-828, 2013.

[18] L. Codecasa and L. Di Rienzo, "Stochastic finite integration technique formulation for electrokinetics," IEEE Trans. Magn., vol. 50, no. 2, p. 7014104, 2014.

[19] B. T. Nguyen, C. Furse, and J. J. Simpson, "A 3d stochastic fdtd model of electromagnetic wave propagation in magnetized ionosphere plasma," IEEE Trans. AP, vol. 63, no. 1, pp. 304-313, 2015.

[20] D. D. Nguyen, "High order fdtd methods for electromagnetic systems in dispersive inhomogeneous media," Ph.D. dissertation, University of Alabama, 2015

[21] N. V. Kantartzis, T. T. Zygiridis, C. S. Antonopoulos, Y. Kanai, and T. D. Tsiboukis, "A generalized domain-decomposition stochastic fdtd technique for complex nanomaterial and graphene structures," IEEE Trans. Magn., vol. 52, no. 3, pp. 1-4, 2016.

[22] J. A. Pereda, L. A. Vielva, V. A, and P. A, "A treatment of magnetized ferrites using the fdtd method," IEEE MGW Lett., vol. 3, no. 5, pp. 136-138, 1993.

[23] J. D. Jackson, Classical electrodynamics. New York: Wiley, 1975. 